\title{
ERRATUM
}

\section{Effect of glycemic index on whole-body substrate oxidation in obese women}

\author{
EO Díaz, JE Galgani, CA Aguirre, IJ Atwater and R Burrows
}

International Journal of Obesity (2005) 29, 879. doi:10.1038/sj.ijo.0802967

Correction to: International Journal of Obesity (2005) 29, 108-114. doi:10.1038/sj.ijo.0802592

Due to a typesetting error, Table 3 appeared incorrectly in the above article. The correct table is shown below.

Table 3 Postprandial whole-body fat and carbohydrate oxidation rate ${ }^{a}$

\begin{tabular}{|c|c|c|}
\hline & High glycemic & Low glycemic \\
\hline \multicolumn{3}{|l|}{ Fasting } \\
\hline $\mathrm{BMR}^{\mathrm{b}}(\mathrm{kJ} /$ day $)$ & $6243 \pm 846$ & $6434 \pm 823$ \\
\hline Fat oxidation (mg/kg FFM/min) & $1.16 \pm 0.39$ & $1.26 \pm 0.32$ \\
\hline $\mathrm{CHO}$ oxidation $(\mathrm{mg} / \mathrm{kg} \mathrm{FFM} / \mathrm{min})$ & $2.54 \pm 0.84$ & $2.51 \pm 0.47$ \\
\hline \multicolumn{3}{|l|}{ Breakfast } \\
\hline $\mathrm{TEE}_{\mathrm{m}}^{\mathrm{c}}(\mathrm{kJ} / \mathrm{min})$ & $6.10 \pm 0.59$ & $6.06 \pm 0.61$ \\
\hline Fat oxidation $(\mathrm{mg} / \mathrm{kg} \mathrm{FFM})^{\mathrm{d}}$ & $329 \pm 103$ & $300 \pm 67$ \\
\hline $\mathrm{CHO}$ oxidation $(\mathrm{mg} / \mathrm{kg} \mathrm{FFM})^{d}$ & $1297 \pm 296$ & $1350 \pm 209$ \\
\hline \multicolumn{3}{|l|}{ Lunch } \\
\hline $\mathrm{TEE}_{\mathrm{m}}^{\mathrm{c}}(\mathrm{kJ} / \mathrm{min})$ & $6.11 \pm 0.52$ & $6.02 \pm 0.56$ \\
\hline Fat oxidation $(\mathrm{mg} / \mathrm{kg} \mathrm{FFM})^{\mathrm{d}}$ & $190 \pm 44$ & $207 \pm 79$ \\
\hline $\mathrm{CHO}$ oxidation $(\mathrm{mg} / \mathrm{kg} \mathrm{FFM})^{\mathrm{d}}$ & $1391 \pm 168$ & $1293 \pm 223$ \\
\hline
\end{tabular}

${ }^{\mathrm{a}}$ Mean \pm s.d. ${ }^{\mathrm{b}}$ Basal metabolic rate. ${ }^{\mathrm{C}}$ Measured total energy expenditure. ${ }^{\mathrm{d}}$ Expressed as $\mathrm{mg} / \mathrm{kg}$ FFM for entire postbreakfast (280 measurements) or postlunch (245 measurements) period. Data were analyzed by $t$-paired test. 